\title{
OPE in Wilson lines with sub-eikonal spin corrections for TMDs and $g_{1}$ structure function
}

\author{
Giovanni Antonio Chirilli,** \\ ${ }^{1}$ Institut für Theoretische Physik, University of Regensburg, D-93040 Regensburg, Germany
}

\begin{abstract}
Low- $x$ evolution of spin-dependent TMDs and spin $g_{1}$ structure function are relevant for the future Electron Ion Collider. To study spin-dynamics at high energies it is necessary to extend the eikonal approximation to include sub-eikonal corrections. I will discuss the Operator Product Expansion (OPE) in terms of Wilson lines with sub-eikonal corrections and derive the sub-eikonal quark propagator.
\end{abstract}

\section{Introduction}

Most of the progress in high-energy QCD has been obtained within the eikonal approximation. The high precision reached by the experiments and the possibility to study spin-dynamics in Deep Inelastic (DIS) scattering at the proposed Electron Ion Collider [1], makes the study of sub-eikonal corrections especially timely.

In order to access the sub-eikonal corrections in high-energy QCD one has to study the sub-eikonal corrections of the propagators of the theory extending, in this way, the Operator Product Expansion to include the sub-eikonal corrections. The result will be relevant for the study of TMDs and $g_{1}$ structure function.

In the eikonal approximation, the operator which describes the DIS scattering amplitude is a trace of two Wilson lines. Its evolution equation with respect to the rapidity of the fields generates the Balitsky-hierarchy [3] which is equivalent to the Jalilian-Marian-Iancu-McLerran-WeigertLeonidov-Kovner(JIMWLK) [6-8] evolution equation thus, they are referred to as B-JIMWLK equation. The Balitsky-Kovchegov (BK) equation $[4,5]$ is the first of the Balitsky hierarchy and it is obtained in the mean field approximation. Its linearization coincides with the BFKL $[9,10]$ equation. As we will see, the sub-eikonal corrections will introduce new operators which we will result in new evolution equations thus providing further information on the QCD dynamics at high energy.

We will start with the derivation of the scalar propagator in the background of a highly boosted gluonic field generated by the target in order to lay down the formalism and the notation used. We then present the result for the quark propagator and make, as an example, a simple application of the OPE with the new derived operators.

\footnotetext{
*e-mail: giovanni.chirilli@ur.de
} 


\section{Scalar propagator}

\subsection{Eikonal approximation}

To reach the high-energy kinematics, we will perform a longitudinal boost of the fields generated by the target-particle in which the projectile particle propagates.

We assume that the projectile-particle is propagating along $p_{1}$ direction, while the target particle is moving along $p_{2}$ direction, where $p_{1}^{\mu}$ and $p_{2}^{\mu}$ are two light-cone vectors such that $p_{1 \mu} p_{2}^{\mu}=p_{1} \cdot p_{2}=\frac{s}{2}$. We can perform a Sudakov decomposition of the momentum any momentum $p^{\mu}$ as $p^{\mu}=\alpha p_{1}^{\mu}+\beta p_{2}^{\mu^{2}}+$ $p_{\perp}^{\mu}$. The light-cone components are defined as $x_{\bullet}=x_{\mu} p_{1}^{\mu}=\sqrt{\frac{s}{2}} x^{-}$and $x_{*}=x_{\mu} p_{2}^{\mu}=\sqrt{\frac{s}{2}} x^{+}$with $x^{ \pm}=\frac{x^{0} \pm x^{3}}{\sqrt{2}}$.

The gauge field $A^{\mu}$ generated by the target, under a boost, gets rescaled by a large parameter $\lambda$ as follows

$$
\begin{aligned}
& A_{\bullet}\left(x_{\bullet}, x_{*}, x_{\perp}\right) \rightarrow \lambda A_{\bullet}\left(\lambda^{-1} x_{\bullet}, \lambda x_{*}, x_{\perp}\right), \\
& A_{*}\left(x_{\bullet}, x_{*}, x_{\perp}\right) \rightarrow \lambda^{-1} A_{*}\left(\lambda^{-1} x_{\bullet}, \lambda x_{*}, x_{\perp}\right), \\
& A_{\perp}\left(x_{\bullet}, x_{*}, x_{\perp}\right) \rightarrow A_{\perp}\left(\lambda^{-1} x_{\bullet}, \lambda x_{*}, x_{\perp}\right) .
\end{aligned}
$$

In Schwinger representation the free scalar propagator is

$$
\left\langle x\left|\frac{i}{p^{2}+i \epsilon}\right| y\right\rangle=i \int d^{4} k \frac{e^{-i k \cdot(x-y)}}{p^{2}+i \epsilon},
$$

with $\langle k \mid x\rangle=e^{i x \cdot k}$. In (2) we used the $\hbar$-inspired notation $d^{4} k \equiv \frac{d^{4} k}{(2 \pi)^{4}}$ and $\delta^{(4)}(x-y)=(2 \pi)^{4} \delta^{(4)}(x-y)$ so that, $\int d^{4} k \delta^{(4)}(x-y)=1$.

In the eikonal approximation, the only component surviving the boost is $A$ • so, $\hat{P}^{2} \simeq \hat{p}^{2}+2 \alpha g \hat{A}$. and we can represent the propagator as a series

$$
\left\langle x\left|\frac{i}{\hat{P}^{2}+i \epsilon}\right| y\right\rangle \simeq\left\langle x\left|\frac{i}{\hat{p}^{2}+i \epsilon}\right| y\right\rangle+g \int d^{4} z\left\langle x\left|\frac{i}{\hat{p}^{2}+i \epsilon}\right| z\right\rangle 2 i \alpha A_{\bullet}\left(z_{*}, z_{\perp}\right)\left\langle z\left|\frac{i}{\hat{p}^{2}+i \epsilon}\right| y\right\rangle+\ldots
$$

In the shock-wave picture (see Fig. 1) relevant for high-energy scattering, we assume that the particle starts and ends its propagation outside the interval in which the field strength tensor is different then zero (see Fig. 1). With this assumption we can rewrite expansion (3) as

$$
\begin{aligned}
\left\langle x\left|\frac{i}{\hat{P}^{2}+i \epsilon}\right| y\right\rangle= & {\left[\int_{0}^{+\infty} \frac{d \alpha}{2 \alpha} \theta\left(x_{*}-y_{*}\right)-\int_{-\infty}^{0} \frac{d \alpha}{2 \alpha} \theta\left(y_{*}-x_{*}\right)\right] e^{-i \alpha\left(x_{\bullet}-y_{\bullet}\right)} \int d^{2} z d^{2} z^{\prime}\left\langle x_{\perp}\left|e^{-i \frac{\hat{p}_{\perp}^{2}}{\alpha s} x_{*}}\right| z_{\perp}\right\rangle } \\
& \times\left\langle z_{\perp}\left|\operatorname{Pexp}\left\{i g \int_{y_{*}}^{x_{*}} d \frac{2}{S} \omega_{*} e^{i \frac{\hat{p}_{\perp}^{2}}{\alpha s} \omega_{*}} A_{\bullet}\left(\omega_{*}\right) e^{-i \frac{\hat{p}_{\perp}^{2}}{\alpha s} \omega_{*}}\right\}\right| z_{\perp}^{\prime}\right\rangle\left\langle z_{\perp}^{\prime}\left|e^{i \frac{\hat{p}_{\perp}^{2}}{\alpha s} y_{*}}\right| y_{\perp}\right\rangle .
\end{aligned}
$$

Eq. (4) has been obtained assuming the commutation relation $[\hat{\alpha}, \hat{A}]=0$ and the fact that the most dominant component of the gauge external field is $A_{\text {. }}$.

Under the longitudinal Lorentz boost, the longitudinal distance traveled by the particle in the external filed is rescaled as $\omega_{*} \rightarrow \frac{1}{\lambda} \omega_{*}$ while the gauge field is rescaled as $A_{\bullet} \rightarrow \lambda A$ • with $\lambda \gg 1$ the boost parameter. So, we can write

$$
e^{i \frac{\hat{p}_{\perp}^{2}}{\alpha s} \omega_{*}} A_{\bullet}\left(\omega_{*}\right) e^{-i \frac{\hat{p}_{\perp}^{2}}{\alpha s} \omega_{*}}=A_{\bullet}\left(\omega_{*}\right)+O\left(\lambda^{0}\right) .
$$


Making use of (5) in propagator (4), we obtain

$$
\begin{aligned}
\left\langle x\left|\frac{i}{\hat{P}^{2}+i \epsilon}\right| y\right\rangle= & {\left[\int_{0}^{+\infty} \frac{d \alpha}{2 \alpha} \theta\left(x_{*}-y_{*}\right)-\int_{-\infty}^{0} \frac{d \alpha}{2 \alpha} \theta\left(y_{*}-x_{*}\right)\right] e^{-i \alpha\left(x_{\bullet}-y_{\bullet}\right)} } \\
& \times \int d^{2} z\left\langle x_{\perp}\left|e^{-i \frac{\hat{p}_{\perp}^{2}}{\alpha s} x_{*}}\right| z_{\perp}\right\rangle\left[x_{*}, y_{*}\right]_{z}\left\langle z_{\perp}\left|e^{i \frac{\hat{p}_{\perp}^{2}}{\alpha s} y_{*}}\right| y_{\perp}\right\rangle
\end{aligned}
$$

where we have defined the gauge link at fixed transverse position $z_{\perp}$ as

$$
\left[x_{*}, y_{*}\right]_{z}=\operatorname{Pexp}\left\{i g \frac{2}{s} \int_{y_{*}}^{x_{*}} d \omega_{*} A_{\bullet}\left(\omega_{*}, z_{\perp}\right)\right\} .
$$

Propagator (6) describes the propagation of the particle in the external field along a straight line. To consider deviation from the straight-line propagation we need to take into account higher order terms in Eq. (5).

Now we can trade the finite gauge link with the infinite Wilson line because, under the infinite boost, the dominant component of the field strength tensor, $F_{\bullet}$, has an infinitesimal thin support in $x_{*}$ coordinate and we assumed that $F_{\bullet i}$ is peaked at the origin while outside the infinitesimal interval $\left[-\epsilon_{*}, \epsilon_{*}\right], F_{\bullet i}=0$ (see Fig. 1). Therefore, in the gauge rotated field $A^{\Omega}$ the gauge field outside the external field is zero and we can trade the gauge link $\left[\epsilon_{*},-\epsilon_{*}\right]$ with the infinite Wilson line $\left[\infty p_{1},-\infty p_{1}\right]$ and obtain

$$
\begin{aligned}
\left\langle x\left|\frac{i}{P^{2}+i \epsilon}\right| y\right\rangle= & {\left[\int_{0}^{+\infty} \frac{d \alpha}{2 \alpha} \theta\left(x_{*}-y_{*}\right)-\int_{-\infty}^{0} \frac{d \alpha}{2 \alpha} \theta\left(y_{*}-x_{*}\right)\right] e^{-i \alpha\left(x_{\bullet}-y_{\bullet}\right)} } \\
& \times \int d^{2} z\left\langle x_{\perp}\left|e^{-i \frac{\hat{p}_{\perp}^{2}}{\alpha s} x_{*}}\right| z_{\perp}\right\rangle U_{z}\left\langle z_{\perp}\left|e^{i \frac{\hat{p}_{\perp}^{2}}{\alpha s} y_{*}}\right| y_{\perp}\right\rangle
\end{aligned}
$$

where we have defined the infinite Wilson line $U_{z}$ at fixed transverse position $z_{\perp}$ as

$$
U_{z} \equiv\left[p_{1} \infty,-p_{1} \infty\right]_{z}=\operatorname{Pexp}\left\{i g \frac{2}{s} \int_{-\infty}^{+\infty} d z_{*} A \cdot\left(\frac{2}{s} p_{1} z_{*}+z_{\perp}\right)\right\} .
$$

Notice that, we can restore $A_{\perp}^{\mu}$ component as a transverse gauge link (see Fig. 1) since it is a pure gauge.

\section{2 sub-eikonal approximation}

In this section we are interested in deriving the sub-eikonal corrections to the scalar propagator Eq. (6). To this end, we consider a background gauge field with all components different than zero, $A_{\mu}^{c l}\left(x_{*}, x_{\perp}\right)=\left(A_{*}^{c l}\left(x_{*}, x_{\perp}\right), A_{\bullet}^{c l}\left(x_{*}, x_{\perp}\right), A_{\perp}^{c l}\left(x_{*}, x_{\perp}\right)\right)$, and we need to identify the most dominant component and sub-dominant one from the operator $\hat{P}^{2}=\left\{\hat{p}_{\perp}^{\mu}, \hat{A}_{\mu}^{\perp}\right\}+\left\{\frac{2}{s} \hat{P}_{\bullet}, \hat{A}_{*}\right\}-g \hat{A}_{\perp}^{2}$.

It can be shown that the term $\left\{\frac{2}{s} \hat{P}_{\bullet}, \hat{A}_{*}\right\}$ is related to the possibility for the fields to depend mildly on $x_{\bullet}$ but it contributes as sub-sub-correction, so we will neglect it and keep considering fields $x_{\bullet}$ independent.

It is convenient to define $\hat{O}=\left\{\hat{p}_{\perp}^{\mu}, \hat{A}_{\mu}^{\perp}\right\}-g \hat{A}_{\perp}^{2}$ so that $\hat{P}^{2}=\hat{p}^{2}+2 g \alpha A \bullet+g \hat{O}$ and the scalar propagator can be written as

$$
\begin{aligned}
\left\langle x\left|\frac{i}{\hat{P}^{2}+i \epsilon}\right| y\right\rangle= & \left\langle x\left|\frac{i}{\hat{p}^{2}+2 \alpha g \hat{A} \bullet+g \hat{O}+i \epsilon}\right| y\right\rangle \\
= & {\left[\int_{0}^{+\infty} \frac{d \alpha}{2 \alpha} \theta\left(x_{*}-y_{*}\right)-\int_{-\infty}^{0} \frac{d \alpha}{2 \alpha} \theta\left(y_{*}-x_{*}\right)\right] e^{-i \alpha\left(x_{\bullet}-y_{\bullet}\right)} } \\
& \times\left\langle x_{\perp}\left|e^{-i \frac{\hat{p}_{\perp}^{2}}{\alpha s} x_{*}} \operatorname{Pexp}\left\{i g \int_{y_{*}}^{x_{*}} d \frac{2}{s} \omega_{*} e^{i \frac{\hat{p}_{\perp}^{2}}{\alpha s} \omega_{*}}\left(\hat{A}_{\bullet}\left(\omega_{*}\right)+\frac{\hat{O}\left(\omega_{*}\right)}{2 \alpha}\right) e^{-i \frac{\hat{p}_{\perp}^{2}}{\alpha s} \omega_{*}}\right\} e^{i \frac{\hat{p}_{\perp}^{2}}{\alpha s} y_{*}}\right| y_{\perp}\right\rangle .
\end{aligned}
$$




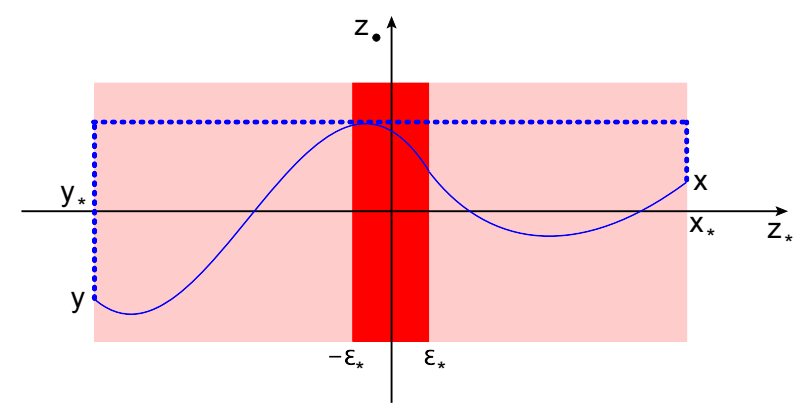

Figure 1. Particle propagating in an external gluonic field. The red strip represents the shock-wave defined in the infinitesimal interval $\left[-\epsilon_{*}, \epsilon_{*}\right]$ in which $F_{\mu \nu} \neq 0$. The light-red areas to the left and to the right of the shock-wave represent the background filed made of pure gage field. The curvy line is the particle's path from point $x$ to point $y$. The dotted lines represent the Wilson lines.

We are now ready to consider the sub-dominant contributions we neglected in the previous section. We observe that

$$
e^{i \frac{\hat{p_{1}^{2}} \alpha \omega_{*}}{\alpha s}}\left(\hat{A}_{\bullet}+\frac{\hat{O}}{2 \alpha}\right) e^{-i \frac{\hat{p}_{\perp}^{2}}{\alpha s} \omega_{*}}=\hat{A}_{\bullet}+\frac{\hat{O}}{2 \alpha}+i \frac{\omega_{*}}{\alpha s}\left[\hat{p}_{\perp}^{2}, \hat{A}_{\bullet}\right]+O\left(\lambda^{-1}\right) .
$$

Using Eq. (11), after some algebra we arrive at

$$
\begin{aligned}
\left\langle x\left|\frac{i}{\hat{P}^{2}+i \epsilon}\right| y\right\rangle= & {\left[\int_{0}^{+\infty} \frac{d \alpha}{2 \alpha} \theta\left(x_{*}-y_{*}\right)-\int_{-\infty}^{0} \frac{d \alpha}{2 \alpha} \theta\left(y_{*}-x_{*}\right)\right] e^{-i \alpha\left(x_{\bullet}-y_{\bullet}\right)} } \\
& \times\left\langle x_{\perp}\right| e^{-i \frac{\hat{p}_{\perp}^{2}}{\alpha s} x_{*}}\left\{\left[x_{*}, y_{*}\right]+\frac{i g}{2 \alpha}\left[\frac{2}{s} x_{*}\left(\left\{P_{i}, A^{i}\left(x_{*}\right)\right\}-g A_{i}\left(x_{*}\right) A^{i}\left(x_{*}\right)\right)\left[x_{*}, y_{*}\right]\right.\right. \\
& -\left[x_{*}, y_{*}\right] \frac{2}{s} y_{*}\left(\left\{P_{i}, A^{i}\left(y_{*}\right)\right\}-g A_{i}\left(y_{*}\right) A^{i}\left(y_{*}\right)\right) \\
& +\int_{y_{*}}^{x_{*}} d \frac{2}{s} \omega_{*}\left(\left\{P^{i},\left[x_{*}, \omega_{*}\right] \frac{2}{s} \omega_{*} F_{i \bullet}\left(\omega_{*}\right)\left[\omega_{*}, y_{*}\right]\right\}\right. \\
& \left.\left.\left.+g \int_{\omega_{*}}^{x_{*}} d \frac{2}{s} \omega_{*}^{\prime} \frac{2}{s}\left(\omega_{*}-\omega_{*}^{\prime}\right)\left[x_{*}, \omega_{*}^{\prime}\right] F^{i} \bullet\left[\omega_{*}^{\prime}, \omega_{*}\right] F_{i \bullet}\left[\omega_{*}, y_{*}\right]\right)\right]\right\} e^{i \frac{\hat{p}_{\perp}^{2}}{\alpha s} y_{*}\left|y_{\perp}\right\rangle+O\left(\lambda^{-2}\right) .}
\end{aligned}
$$

As we will see in the next section, propagator (12) represent an intermediate useful step in order to obtain the quark propagator with sub-eikonal corrections.

\section{Quark propagator with sub-eikonal corrections}

The quark propagator in an external field in Schwinger notation is

$$
\left\langle x\left|\frac{i}{\hat{P}+i \epsilon}\right| y\right\rangle=\left\langle x\left|\hat{P} \frac{i}{p^{2}+2 \alpha g A_{\bullet}+g B+i \frac{2}{s} g F_{\bullet i} \not p_{2} \gamma^{i}+i \epsilon}\right| y\right\rangle
$$

where we have defined $B \equiv O+\frac{4}{s^{2}} g F_{\bullet *} \sigma_{* \bullet}+\frac{g}{2} F_{i j} \sigma^{i j}$. 
To get the sub eikonal corrections to the quark propagator we have to expand (13) in terms of the sub-dominant contribution and repeat similar steps that we performed to get the sub-eikonal terms for the scalar propagator. Expanding we have

$$
\begin{aligned}
\left\langle x\left|\hat{P} \frac{i}{P^{2}+\frac{g}{2} F_{\mu \nu} \sigma^{\mu \nu}}\right| y\right\rangle= & \langle x| \hat{P}\left[\frac{i}{P^{2}}-\frac{i}{P^{2}}\left(i g \frac{2}{s} F_{i \bullet} \gamma^{i} \not p_{2}+g \mathcal{B}_{1}\right) \frac{1}{P^{2}}\right. \\
& \left.+\frac{i}{P^{2}}\left(i g \frac{2}{S} F_{i \bullet} \gamma^{i} \not p_{2}+g \mathcal{B}_{1}\right) \frac{1}{P^{2}}\left(i g \frac{2}{S} F_{i \bullet} \gamma^{i} \not p_{2}+g \mathcal{B}_{1}\right) \frac{1}{P^{2}}\right]|y\rangle
\end{aligned}
$$

where we defined $\mathcal{B}_{1}=\frac{4}{s^{2}} F_{\bullet *} \sigma_{* \bullet}+\frac{1}{2} \sigma_{i j} F^{i j}$.

We would like to obtain a gauge invariant expression of Eq. (14) so, after some algebra (see ref. [11] for the details of the derivation) we arrive at

$$
\begin{aligned}
\left\langle x\left|\frac{i}{\hat{p}+i \epsilon}\right| y\right\rangle= & {\left[\int_{0}^{+\infty} \frac{d \alpha}{2 \alpha} \theta\left(x_{*}-y_{*}\right)-\int_{-\infty}^{0} \frac{d \alpha}{2 \alpha} \theta\left(y_{*}-x_{*}\right)\right] e^{-i \alpha\left(x_{\bullet}-y_{\bullet}\right)} \frac{1}{\alpha s} } \\
& \times\left\langle x_{\perp}\right| e^{-i \frac{\hat{p}_{\perp}^{2}}{\alpha s} x_{*}}\left\{\hat{p p} \not p_{2}\left[x_{*}, y_{*}\right] \hat{p}+\hat{p} \not p_{2} \hat{O}_{1}\left(x_{*}, y_{*} ; p_{\perp}\right) \hat{\not p}\right. \\
& \left.+\hat{p}_{p} p_{2} \frac{1}{2} \hat{O}_{2}\left(x_{*}, y_{*} ; p_{\perp}\right)-\frac{1}{2} \hat{O}_{2}\left(x_{*}, y_{*} ; p_{\perp}\right) \not p_{2} \hat{p p}\right\} e^{i \frac{\hat{p}_{\perp}^{2}}{\alpha s} y_{*}}\left|y_{\perp}\right\rangle+O\left(\lambda^{-2}\right) .
\end{aligned}
$$

where we have defined the operators

$$
\begin{aligned}
\hat{O}_{1}\left(x_{*}, y_{*} ; p_{\perp}\right)= & \frac{i g}{2 \alpha} \int_{y_{*}}^{x_{*}} d \frac{2}{s} \omega_{*}\left(\left[x_{*}, \omega_{*}\right] \frac{1}{2} \sigma^{i j} F_{i j}\left[\omega_{*}, y_{*}\right]+\left\{\hat{p}^{i},\left[x_{*}, \omega_{*}\right] \frac{2}{S} \omega_{*} F_{i \bullet}\left(\omega_{*}\right)\left[\omega_{*}, y_{*}\right]\right\}\right. \\
& \left.+g \int_{\omega_{*}}^{x_{*}} d \frac{2}{S} \omega_{*}^{\prime} \frac{2}{S}\left(\omega_{*}-\omega_{*}^{\prime}\right)\left[x_{*}, \omega_{*}^{\prime}\right] F^{i} \cdot\left[\omega_{*}^{\prime}, \omega_{*}\right] F_{i \bullet}\left[\omega_{*}, y_{*}\right]\right),
\end{aligned}
$$

and

$$
\begin{aligned}
\hat{O}_{2}\left(x_{*}, y_{*} ; p_{\perp}\right)= & \frac{i g}{2 \alpha} \int_{y_{*}}^{x_{*}} d \frac{2}{S} \omega_{*}\left[\left[x_{*}, \omega_{*}\right] \frac{1}{4}\left(i \mathfrak{D}_{k} F_{i j}\right)\left\{\sigma^{i j}, \gamma^{k}\right\}\left[\omega_{*}, y_{*}\right]+\left\{\hat{p}_{k},\left[x_{*}, \omega_{*}\right] i F_{k j} \gamma^{j}\left[\omega_{*}, y_{*}\right]\right\}\right. \\
& +\left[x_{*}, \omega_{*}\right] i F_{k j} \gamma^{j}\left(i \mathfrak{D}^{k}\left[\omega_{*}, y_{*}\right]\right)-\left(i \mathfrak{D}^{k}\left[x_{*}, \omega_{*}\right]\right) i F_{k j} \gamma^{j}\left[\omega_{*}, y_{*}\right] \\
& +\left(\hat{\alpha} \not p_{1}-\hat{p}_{\perp}\right)\left[x_{*}, \omega_{*}\right] i \frac{2}{S} F_{\bullet *}\left[\omega_{*}, y_{*}\right]+\left(i \not D_{\perp}\left[x_{*}, \omega_{*}\right]\right) i \frac{2}{S} F_{\bullet *}\left[\omega_{*}, y_{*}\right] \\
& \left.-\left[x_{*}, \omega_{*}\right] i \frac{2}{S} F_{\bullet *}\left(i D_{\perp}\left[\omega_{*}, y_{*}\right]\right)\right]
\end{aligned}
$$

From propagator (15), given an observable in terms of operators, one may apply the high-energy OPE and obtain sub-eikonal corrections to the scattering amplitude as a convolution of coefficient functions and matrix elements of Wilson lines with the insertion of the new operators appearing in Eqs. (16) and (17).

\section{OPE in Wilson lines with sub-eikonal corrections}

As an example, let us apply the high-energy OPE with sub-eikonal corrections to DIS process described by the two electromagnetic currents evaluated in the nucleon (or nucleus) state (See ref. [2] 
for a review on Wilson lines formalism at high energy). For simplicity, let us consider only the subeikonal term given by the operator $\frac{1}{2} F_{i j} \sigma^{i j}$. We have

$$
\begin{gathered}
\left\langle P, S\left|\bar{\psi}(x) \gamma^{\mu} \psi(x) \bar{\psi}(y) \gamma^{v} \psi(y)\right| P, S\right\rangle \stackrel{x_{*}>0>y_{*}}{=} \int d^{2} z_{1} d^{2} z_{2} \frac{\operatorname{tr}\left\{X_{1} p_{2} Y_{1} \gamma^{v} Y_{2} \not p_{2} X_{2} \gamma^{\mu}\right\}}{4 \pi^{6} x_{*}^{4} y_{*}^{4} \mathcal{Z}_{1}^{3} Z_{2}^{3}}\left\langle P, S\left|\operatorname{tr}\left\{U_{z_{1}} U_{z_{2}}^{\dagger}\right\}\right| P, S\right\rangle \\
-g \int d^{2} z_{1} d^{2} z_{2} \frac{\operatorname{tr}\left\{X_{1} \not p_{2} Y_{1} \gamma^{\nu} Y_{2} \not p_{2} \sigma^{i j} X_{2} \gamma^{\mu}\right\}}{64 \pi^{6} x_{*}^{4} y_{*}^{4} \mathcal{Z}_{1}^{3} Z_{2}^{2}} \int_{y_{*}}^{x_{*}} d \omega_{*}\left[\left\langle P, S\left|\operatorname{tr}\left\{U_{z_{1}}\left[-\infty p_{1}, \omega_{*}\right]_{z_{2}} F_{i j}\left[\omega_{*},+\infty p_{1}\right]_{z_{2}}\right\}\right| P, S\right\rangle\right. \\
\left.+\left\langle P, S\left|\operatorname{tr}\left\{\left[+\infty p_{1}, \omega_{*}\right]_{z_{2}} F_{i j}\left[\omega_{*},-\infty p_{1}\right]_{z_{2}} U_{z_{1}}^{\dagger}\right\}\right| P, S\right\rangle\right]
\end{gathered}
$$

where we have defined

$$
\mathcal{Z}_{i} \equiv \frac{\left(x-z_{i}\right)_{\perp}^{2}}{x_{*}}-\frac{\left(y-z_{i}\right)_{\perp}^{2}}{y_{*}}-\frac{4}{s}\left(x_{\bullet}-y_{\bullet}\right)
$$

and $X_{i} \equiv x-z_{i}$, and $\quad Y_{i} \equiv y-z_{i}$.

The first term after equal sign in Eq. (18) is given by the Leading Order (LO) Impact Factor (the coefficient function of an OPE), which has been known for more than 40 years, and matrix element of two Wilson lines whose evolution equation with respect to the rapidity parameter gives the BK equation [3-5] or the B-JIMWLK equation [6-8] according to whether we apply the mean-field approximation or not.

The second term after equal sign in Eq. (18) is the new term in the OPE which comes from the sub-eikonal corrections in the quark propagator. We would have more terms in OPE (18), had we considered all terms given in operators $\hat{O}_{1}$ and $\hat{O}_{2}$.

Let us consider the evolution equation of the Wilson line with the insertion of the operator $\frac{1}{2} F_{i j} \sigma^{i j}$. The one loop diagram are given in Fig. (2) and the evolution equation is

$$
\begin{aligned}
& \left.\int_{-\infty}^{+\infty} d \omega_{*} \operatorname{tr}\left\{[\infty,-\infty]_{z}\left[-\infty, \omega_{*}\right]_{z^{\prime}} g F_{i j}\left(\omega_{*}\right)\left[\omega_{*}, \infty\right]_{z^{\prime}}\right\}\right\rangle_{\mathrm{BK}-\text { type }} \\
& =\frac{\alpha_{s}}{2 \pi^{2}} \int_{-\infty}^{+\infty} d \omega_{*} \int_{0}^{+\infty} \frac{d \alpha}{\alpha} \int d^{2} \omega \frac{\left(z-z^{\prime}\right)_{\perp}^{2}}{(z-\omega)_{\perp}^{2}\left(z^{\prime}-\omega\right)_{\perp}^{2}} \\
& \times\left[\operatorname{tr}\left\{U_{z} U_{\omega}^{\dagger}\right\} \operatorname{tr}\left\{U_{\omega}\left[-\infty, \omega_{*}\right]_{z^{\prime}} g F_{i j}\left(\omega_{*}, z_{\perp}^{\prime}\right)\left[\omega_{*}, \infty\right]_{z^{\prime}}\right\}-N_{c} \operatorname{tr}\left\{U_{z}\left[-\infty, \omega_{*}\right]_{z^{\prime}} g F_{i j}\left(\omega_{*}, z_{\perp}^{\prime}\right)\left[\omega_{*}, \infty\right]_{z^{\prime}}\right\}\right]
\end{aligned}
$$

We observe that, besides the rapidity divergence given by $\int_{0}^{\infty} \frac{d \alpha}{\alpha}$ we have an extra divergence for $\omega \rightarrow z_{\perp}^{\prime}$. The evolution equation (20) indeed, resum $\left(\alpha_{s} \ln ^{2} \frac{1}{x_{B}}\right)$, which is the type of resummation one finds in polarized DIS $[12,13]$.

\section{Conclusions}

We have presented the quark propagator with sub-eikonal corrections in high-energy QCD. Such result is relevant for the description, for example, of spin-dynamics at high-energy.

Most of the results obtained in the high-energy QCD have been obtained within the eikonal approximation. Because of the high-precision reached by the experiments and the possibility to access spin-dynamics at the proposed Electron Ion Collider (EIC), the sub-eikonal corrections become relevant.

To obtain information coming from sub-eikonal corrections, we have derived the quark propagator, which is the main result presented here. It is given in Eq. (15) and the new obtained operators are given in Eq. (16) and (17). 


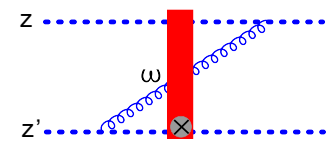

a)

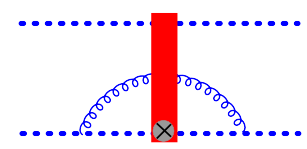

c)

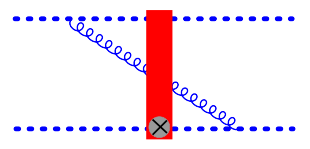

b)

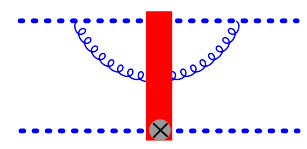

d)

Figure 2. BK-type diagrams for the operator give in Eq. (20)

As an example of the OPE with sub-eikonal corrections, we have considered the sub-eikonal correction described by the operator $\frac{1}{2} F_{i j} \sigma^{i j}$, and have obtained its evolution equation with respect to the rapidity parameter. We noticed that, besides the usual rapidity divergence, which is related to the resummation of $\alpha_{s} \ln \frac{1}{x_{B}}$, one has an extra divergence due to the presence of the operator $\frac{1}{2} F_{i j} \sigma^{i j}$. This new divergence, together with the rapidity divergence, makes up the double Log divergence which is characteristic of the spin-dynamics at high energy.

\section{References}

[1] A. Accardi et al., Eur. Phys. J. A 52 (2016) no.9, 268 doi:10.1140/epja/i2016-16268-9 [arXiv:1212.1701 [nucl-ex]].

[2] I. Balitsky, "High-Energy QCD and Wilson Lines", In *Shifman, M. (ed.): At the frontier of particle physics, vol. 2*, p. 1237-1342 (World Scientific, Singapore, 2001).

[3] I. Balitsky, Nucl. Phys. B 463 (1996) 99 doi:10.1016/0550-3213(95)00638-9.

[4] Y. V. Kovchegov, Small-x $F_{2}$ structure function of a nucleus including multiple pomeron exchanges, Phys. Rev. D60 (1999) 034008,

[5] Y. V. Kovchegov, Unitarization of the BFKL pomeron on a nucleus, Phys. Rev. D61 (2000) 074018,

[6] J. Jalilian-Marian, A. Kovner and H. Weigert, Phys. Rev. D 59, 014015 (1998) doi:10.1103/PhysRevD.59.014015 [hep-ph/9709432].

[7] J. Jalilian-Marian, A. Kovner, A. Leonidov and H. Weigert, Phys. Rev. D 59, 014014 (1998) doi:10.1103/PhysRevD.59.014014 [hep-ph/9706377].

[8] E. Iancu, A. Leonidov and L. D. McLerran, Nucl. Phys. A 692 (2001) 583 doi:10.1016/S03759474(01)00642-X [hep-ph/0011241].

[9] E. A. Kuraev, L. N. Lipatov and V. S. Fadin, The Pomeranchuk singlularity in non-Abelian gauge theories, Sov. Phys. JETP 45 (1977) 199-204.

[10] I. Balitsky and L. Lipatov, The Pomeranchuk Singularity in Quantum Chromodynamics, Sov.J.Nucl.Phys. 28 (1978) 822-829.

[11] G. A. Chirilli, arXiv:1807.11435 [hep-ph].

[12] J. Bartels, B. I. Ermolaev and M. G. Ryskin, Z. Phys. C 70 (1996) 273 [hep-ph/9507271].

[13] J. Bartels, B. I. Ermolaev and M. G. Ryskin, Z. Phys. C 72 (1996) 627 doi:10.1007/s002880050285, 10.1007/BF02909194 [hep-ph/9603204]. 